\title{
The large-scale diffuse radio emission in A781
}

\author{
F. Govoni ${ }^{1}$, M. Murgia ${ }^{1}$, G. Giovannini ${ }^{2,3}$, V. Vacca ${ }^{1,4}$, and A. Bonafede ${ }^{5,3}$ \\ 1 INAF - Osservatorio Astronomico di Cagliari, Strada 54, Loc. Poggio dei Pini, 09012 Capoterra (Ca), Italy \\ e-mail: fgovoni@ca.astro.it \\ 2 Dipartimento di Astronomia, Università degli Studi di Bologna, via Ranzani 1, 40127 Bologna, Italy \\ 3 INAF - Istituto di Radioastronomia, via P. Gobetti 101, 40129 Bologna, Italy \\ 4 Dipartimento di Fisica, Università degli Studi di Cagliari, Cittadella Universitaria, 09042 Monserrato (CA), Italy \\ 5 Jacobs University of Bremen, Campus Ring 1, 28759 Bremen, Germany
}

Received 2 November 2010 / Accepted 4 February 2011

\section{ABSTRACT}

Context. A781 belongs to a complex system characterized by extended X-ray emissions that may form part of line of clusters of galaxies along a filament.

Aims. The aim of this work is to investigate the possible presence of extended, diffuse synchrotron radio emission connected to the intra-cluster medium of A781.

Methods. We studied the radio continuum emission and the spectral index properties in the proximity of A781 by analyzing archival Very Large Array observations at 1400 and $325 \mathrm{MHz}$.

Results. The main cluster of the system is permeated by diffuse low-surface brightness radio emission which is classified as a radio halo because it is located close to the center. The diffuse emission presents the typical extension and radio power of the other halos known in the literature. Interestingly, the radio halo appears to be linked to a peripheral patch that was previously found in the literature. The spectrum of this peripheral emission shows a radial steepening, which may confirm that this source is indeed a cluster relic.

Key words. galaxies: clusters: general - galaxies: clusters: individual: A781 - magnetic fields - large-scale structure of Universe

\section{Introduction}

An ever increasing number of galaxy clusters exhibits at its center an Mpc-scale synchrotron radio halo. These elusive sources are characterized by a low-surface brightness $\left(\sim 1 \mu \mathrm{Jy} / \operatorname{arcsec}^{2}\right.$ at $1400 \mathrm{MHz})$ and a steep-spectrum ${ }^{1}(\alpha>1)$. They are among the best evidence for the presence of relativistic electrons and magnetic fields in the intra-cluster medium.

In the last decade several projects aimed at detecting new radio halos have been performed (e.g. Giovannini et al. 1999; Giovannini \& Feretti 2000; Kempner \& Sarazin 2001; Govoni et al. 2001a; Bacchi et al. 2003; Venturi et al. 2007, 2008; van Weeren et al. 2009; Rudnick \& Lemmerman 2009; Giovannini et al. 2009). The investigation of the physical properties of radio halos and their hosting environments have improved our knowledge and led to the formulation of possible scenarios of their formation, which are still a matter of debate (e.g. Brunetti et al. 2009; Enßlin et al. 2011). The radio halo morphology is often very similar to the X-ray-emitting thermal intra-cluster medium (e.g. Govoni et al. 2001b; Feretti et al. 2001; Giacintucci et al. 2005). Furthermore, radio halos are preferentially found in clusters that show evidence of merger activity (e.g. Buote 2001; Schuecker et al. 2001; Govoni et al. 2004; Cassano et al. 2010), which suggests a connection between the origin of radio halos and gravitational processes of cluster formation, although a one-to-one association between merging clusters and radio halos is not supported by present observations. It is thus fundamental in this context to better investigate the radio emission from merging and $\mathrm{X}$-ray luminous galaxy clusters.
As part of an ongoing program aimed at investigating the presence of halo emission in complex X-ray cluster systems, we recently found (Murgia et al. 2010) the first example of a double radio halo in the close pair of galaxy clusters A399 and A401. Motivated by the above discovery, we analyzed archival Very Large Array (VLA) radio observations of A781. This is an exceptional system, because combined X-ray and weak-lensing analyses (Wittman et al. 2006; Sehgal et al. 2008) indicate that A781 is really a complex of several clusters.

In this work we show how this system appears at the radio wavelengths. The paper is organized as follows: in Sect. 2 we describe the properties of the cluster. In Sect. 3 we present the radio observations and the data reduction. In Sect. 4 we show the results of the total intensity and spectral index images. Finally, we draw the conclusions in Sect. 5.

Throughout this paper we assume a $\Lambda \mathrm{CDM}$ cosmology with $H_{0}=71 \mathrm{~km} \mathrm{~s}^{-1} \mathrm{Mpc}^{-1}, \Omega_{\mathrm{m}}=0.27$, and $\Omega_{\Lambda}=0.73$. At the distance of A781 ( $z=0.3004$, Geller et al. 2010), $1^{\prime \prime}$ corresponds to $4.4 \mathrm{kpc}$.

\section{The A781 system}

A781 belongs to a complex system that is characterized by extended X-ray sources that may form part of a line of clusters along a filament (e.g. Jeltema et al. 2005). The X-ray surface brightness distribution of the system is shown in Fig. 1, where we present an image in the $0.2-12 \mathrm{keV}$ band obtained with a recent XMM-Newton (MOS1+MOS2) exposure time of $80 \mathrm{ks}$ (Obs. id: 0401170101). As previously pointed out by Sehgal et al. (2008), the X-ray image reveals that A781 consists of a large main ("Main") cluster connected to a sub-cluster. In 
Table 1. Details of the VLA observations.

\begin{tabular}{lccccclcc}
\hline \hline Source & $\begin{array}{c}\text { RA } \\
(\mathrm{J} 2000)\end{array}$ & $\begin{array}{c}\text { Dec } \\
(\mathrm{J} 2000)\end{array}$ & $\begin{array}{c}\text { Frequency } \\
(\mathrm{MHz})\end{array}$ & $\begin{array}{c}\text { Bandwidth } \\
(\mathrm{MHz})\end{array}$ & Conf. & $\begin{array}{l}\text { Time } \\
(\mathrm{min})\end{array}$ & Date & Program \\
\hline A781 & 092023.7 & +303109.0 & $1365 / 1435$ & $7 \times 3.125$ & $\mathrm{~A}$ & 30 & $1994-04-19$ & AB699 \\
& 092022.6 & +303120.6 & $1452 / 1502$ & 25 & $\mathrm{C}$ & 8 & $1984-05-04$ & AO048 \\
& 092023.7 & +303109.0 & $1365 / 1435$ & $7 \times 3.125$ & $\mathrm{D}$ & 15 & $1995-03-15$ & AM469 \\
& 091436.0 & +294452.0 & $327.5 / 321.6$ & $64 \times 0.048$ & $\mathrm{~B}$ & 49 & $2005-05-21$ & AD509 \\
& 091436.0 & +294452.0 & $327.5 / 321.6$ & $64 \times 0.048$ & $\mathrm{C}$ & 183,17 & $2005-09-29,10-03$ & AD509 \\
& 091436.0 & +294452.0 & $327.5 / 321.6$ & $64 \times 0.048$ & $\mathrm{D}$ & $88,96,20$ & $2005-12-04,12-09,12-12$ & AD509 \\
\hline
\end{tabular}

Notes. Column 1: source; Cols. 2, 3: pointing position (RA, Dec); Col. 4: observing frequency; Col. 5: observing bandwidth; Col. 6: VLA configuration; Col. 7: time on source; Col. 8: dates of observation; Col. 9: VLA program.

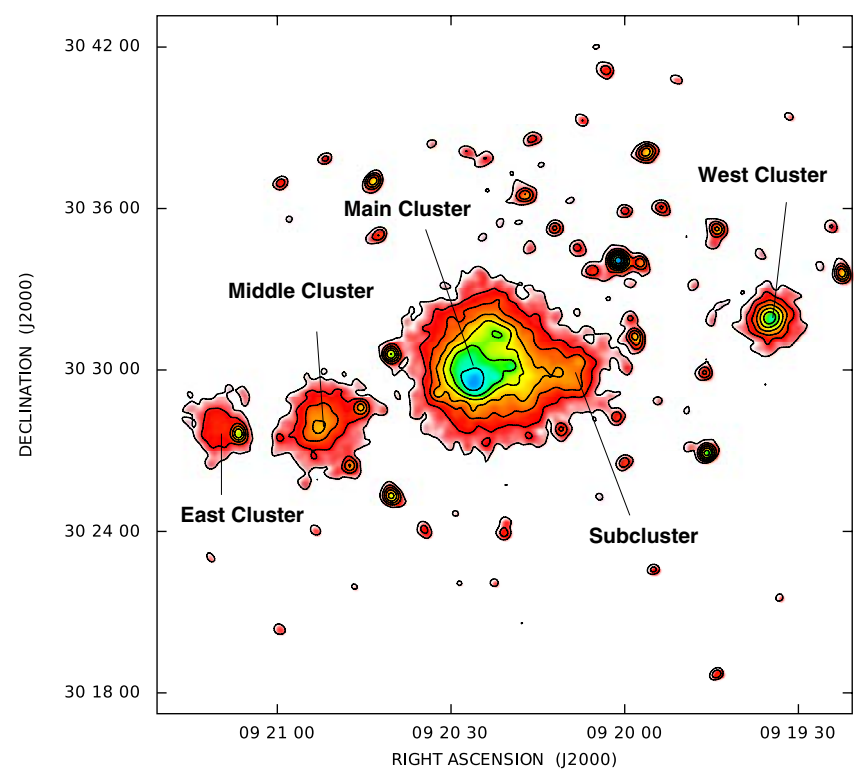

Fig. 1. XMM-Newton image, in the $0.2-12 \mathrm{keV}$ band, of the A781 cluster complex. The X-ray image was convolved with a Gaussian of $\sigma=8^{\prime \prime}$. The first contour level is drawn at $3 \mathrm{cts} /$ Skypixel $(1$ Skypixel $=$ $\left.4^{\prime \prime}\right)$ and the rest are spaced by a factor of $\sqrt{2}$. The clusters are labeled as in Sehgal et al. (2008).

addition, two smaller clusters to its east ("Middle" and "East"), and one to its west ("West") are visible at a relatively small separation on the sky. The X-ray and weak-lensing analysis by Sehgal et al. (2008) suggests that the "Main" cluster may be undergoing a merger with the "Subcluster" to the southwest, while the other clusters are fairly relaxed. The redshift of the "Middle" cluster $(z=0.2915)$ is quite similar to that of the "Main" cluster $(z=0.3004)$. In contrast, the "East" and the "West" clusters have different redshifts ( $z=0.4265$ and $z=0.4273$ respectively; Geller et al. 2010), therefore they are not related to the main cluster of the system.

The A781 system was observed with the Giant Metrewave Radio Telescope at $610 \mathrm{MHz}$ by Venturi et al. (2008). An interesting peripheral patch of diffuse emission, with no obvious optical counterpart, peaking at RA $(J 2000)=09^{\mathrm{h}} 20^{\mathrm{m}} 32.2^{\mathrm{s}}$ $\operatorname{Dec}(\mathrm{J} 2000)=30^{\circ} 27^{\prime} 34.2^{\prime \prime}$ has been detected, and the authors suggested that it might be a relic source, but no hint of radio halo emission was found.

The presence of diffuse cluster-scale radio emission close to the "Main" cluster X-ray centroid, indicative of a radio halo, was suggested by Rudnick \& Lemmerman (2009), by reprocessing radio images from the Westerbork Northern Sky Survey (WENSS, Rengelink et al. 1997) at $327 \mathrm{MHz}$.

\section{VLA observations and data reduction}

Because of the peculiar X-ray morphology of this complex system and because of previous radio results, we investigated the radio continuum emission in the proximity of the A781 system at 1400 and $325 \mathrm{MHz}$. The details of the radio observations are given in Table 1. Calibration and imaging were performed with the NRAO Astronomical Image Processing System (AIPS) package.

\subsection{Observations at $1400 \mathrm{MHz}$}

We analyzed archival VLA observations at $1400 \mathrm{MHz}$ in A, C, and $\mathrm{D}$ configuration. The data were calibrated in phase and amplitude. Data editing was made to excise radio frequency interferences (RFI). In the A and D configuration the observations were made in spectral line mode, therefore the data were calibrated in bandpass and the data editing was made channel by channel. Surface brightness images were produced following the standard procedures: Fourier-Transform, Clean, and Restore implemented in the AIPS task IMAGR. We used the Multiscale CLEAN (see e.g. Greisen et al. 2009), an extension of the classical Clean algorithm, implemented in the task IMAGR. We averaged the two IFs (and the seven channels in the D and A configuration) together in the gridding process under IMAGR. Self-calibration (phase) was performed to increase the dynamic range and sensitivity of the radio images.

A polarization sensitive image has been produced by using the $\mathrm{C}$ configuration data-set (the spectral line mode of the A and $\mathrm{D}$ configuration is not suitable for this purpose).

\subsection{Observations at $325 \mathrm{MHz}$}

There are no pointed low-frequency VLA observations of A781. However, the cluster falls within the field of view (at a distance of $\simeq 1.5^{\circ}$ from the pointing) of the project AD509, which consists of archival observations at $325 \mathrm{MHz}$ in $\mathrm{B}, \mathrm{C}$, and D configuration. The data were collected in spectral line mode with a total bandwidth of $3.1 \mathrm{MHz}$, subdivided into 64 spectral channels. The flux density scale and the bandpass were calibrated by using 3C 48 (3C 147 in B configuration). The source $0909+428$ was used as phase calibrator. Task FLGIT was applied to automatically remove data with strong RFI (about $20-30 \%$ of the data were typically flagged). The data were averaged to 10 channels (with a width of $\simeq 0.3 \mathrm{MHz}$ ), keeping the effects of bandwidth smearing under control. Low-level residual RFI were carefully removed from the 10 channels data-set by visual inspection and finally the data were extracted with the task SPLIT, and imaged. We processed the data-sets separately by applying several cycles of imaging and self-calibration to remove residual phase variations. All data-sets were then combined with the task DBCON, and we performed a final self-calibration run. The area 

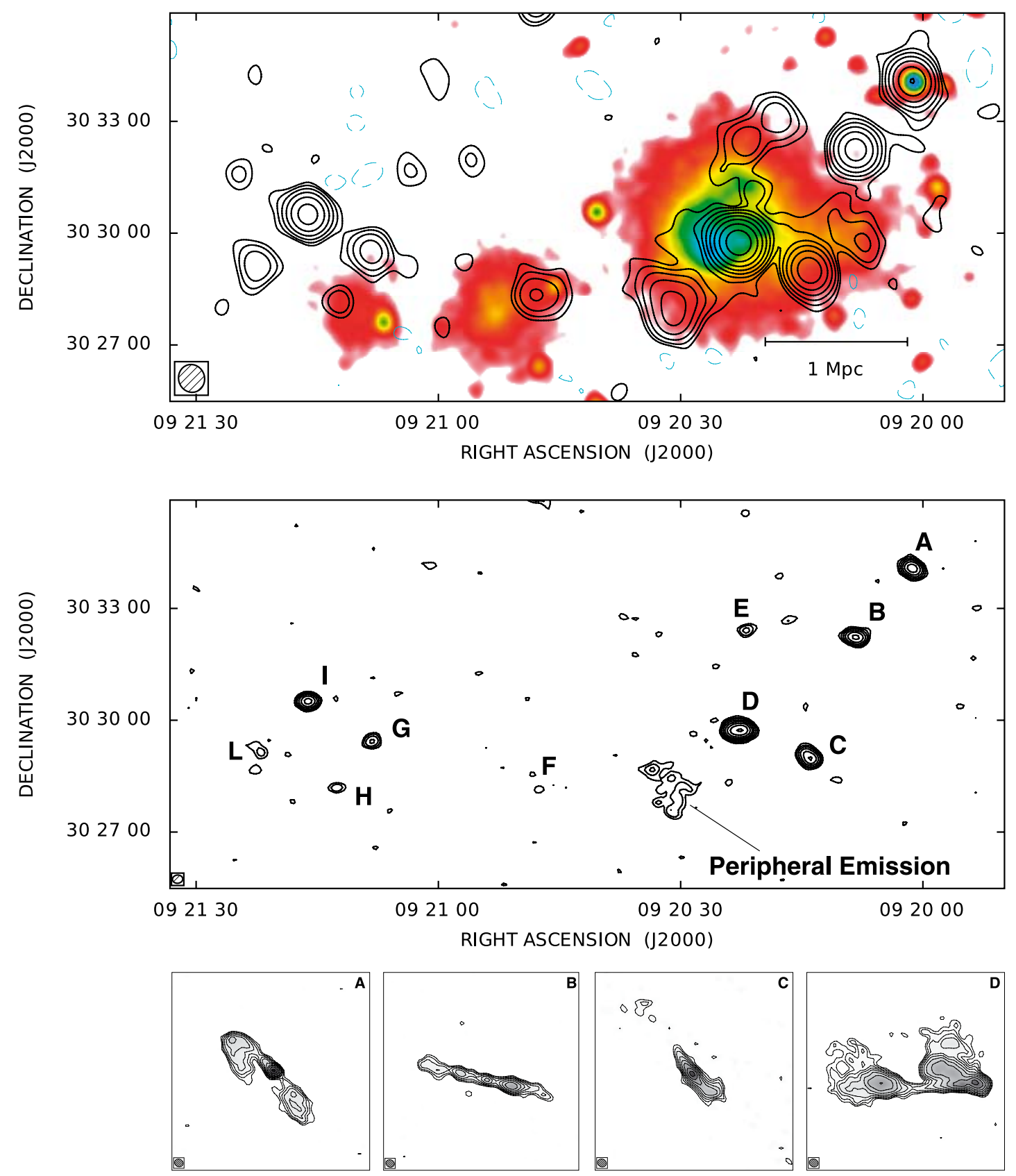

Fig. 2. Top: total intensity radio contours of the A781 system at $1400 \mathrm{MHz}$ with the VLA in D configuration. The image has a $F W H M$ of 41.6" $\times 44.8^{\prime \prime}\left(\mathrm{PA}=16.6^{\circ}\right)$. The contour levels are drawn at -0.3 (thin-dashed lines), $0.3 \mathrm{mJy}$ beam $^{-1}$, and the rest are spaced by a factor of 2 . The sensitivity $(1-\sigma)$ is $0.1 \mathrm{mJy}_{\text {beam }}{ }^{-1}$. Total intensity radio contours are overlaid on the XMM X-ray image in the $0.2-12 \mathrm{keV}$ band. The X-ray image was convolved with a Gaussian of $\sigma=8^{\prime \prime}$. Middle: total intensity radio contours at $1400 \mathrm{MHz}$ with the VLA in C configuration. The image has a $F W H M$ of $13.2^{\prime \prime} \times 16.5^{\prime \prime}\left(\mathrm{PA}=-84.9^{\circ}\right)$. The first contour level is drawn at $0.27 \mathrm{mJy}^{-1} \mathrm{bem}^{-1}$ and the rest are spaced by a factor of 2 . The sensitivity $(1-\sigma)$ is $0.09 \mathrm{mJy}_{\text {beam }}{ }^{-1}$. Bottom: total intensity radio contours of the discrete sources A, B, C, and D at $1400 \mathrm{MHz}$ with the VLA in A configuration. The images have a $F W H M$ of $1.2^{\prime \prime} \times 1.4^{\prime \prime}\left(\mathrm{PA}=67.3^{\circ}\right)$. The sensitivity $(1-\sigma)$ is $0.05 \mathrm{mJy} \mathrm{beam}^{-1}$. The first contour level is drawn at $0.15 \mathrm{mJy}^{-1}$ beam $^{-1}$ and the rest are spaced by a factor of $\sqrt{2}$. The field of view of the images is $30^{\prime \prime}$.

was imaged in facets (Cornwell \& Perley 1992) of slightly overlapping fields to account for the non-coplanarity of the incoming wavefront within the large primary beam of $\sim 3^{\circ}$. The deconvolution was performed with a Multi-scale CLEAN.

\section{Results}

We analyze the results of the VLA observations with a particular emphasis on the analysis of the radio properties of the large-scale diffuse cluster emission.

\subsection{Cluster radio emission at $1400 \mathrm{MHz}$}

The radio iso-contours at $1400 \mathrm{MHz}$ of the "Main", "Middle", and "East" cluster of the system are shown in the top panel of Fig. 2. This image was obtained with the VLA in D configuration and has a $F W H M$ beam of $41.6^{\prime \prime} \times 44.8^{\prime \prime}$. To compare the radio and $\mathrm{X}$-ray cluster emission, the radio iso-contours are overlaid on the XMM-Newton image presented in Fig. 1. We find that the central region of the "Main" cluster is permeated by diffuse low-surface brightness emission, which we classified as a radio halo. The radio halo appears to be linked to the brighter 

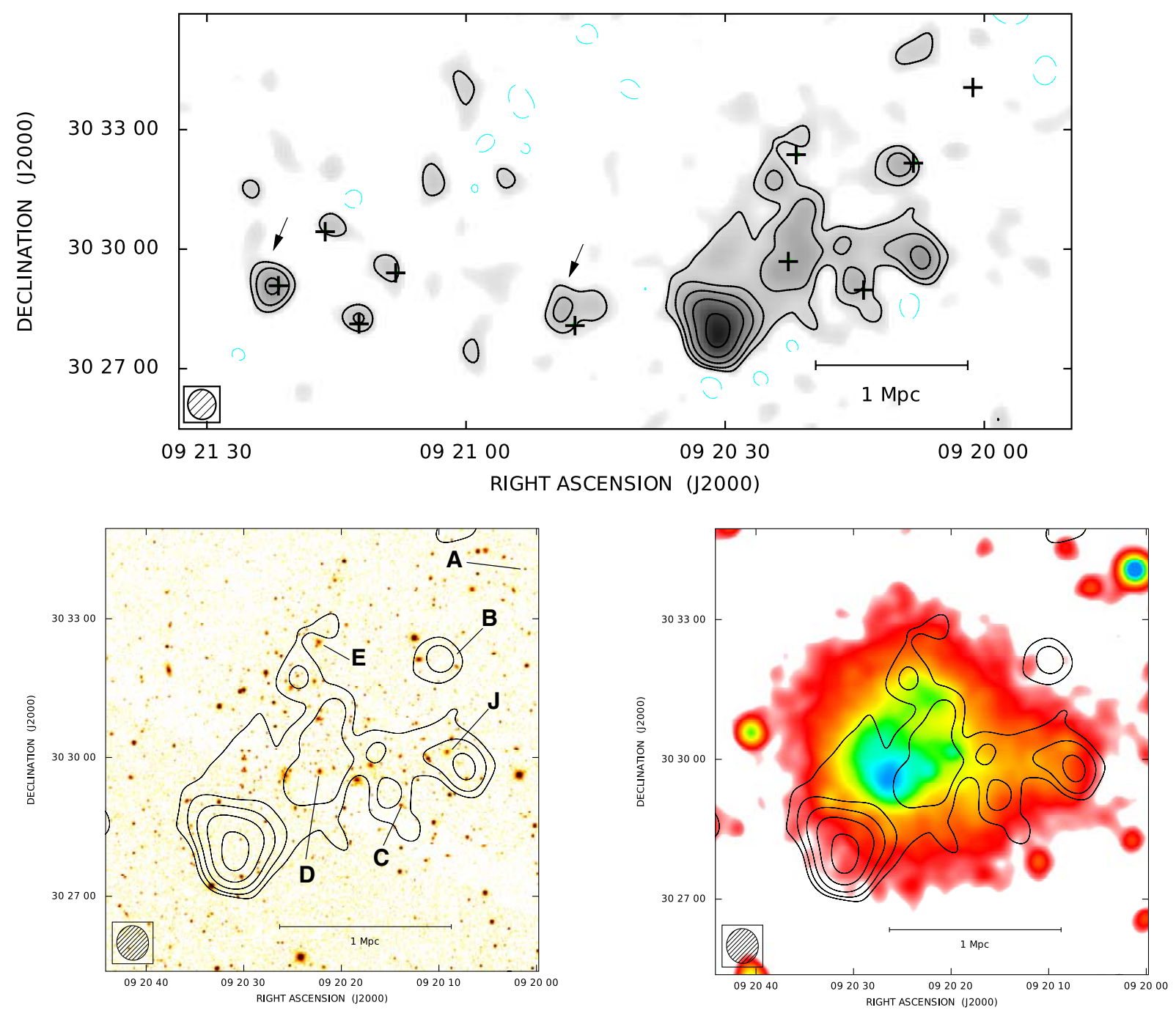

Fig. 3. Top: total intensity radio contours at $1400 \mathrm{MHz}$ with the VLA in D configuration after subtraction of discrete sources. The image has a $F W H M$ of $42^{\prime \prime} \times 45^{\prime \prime}\left(\mathrm{PA}=10.3^{\circ}\right)$. The contour levels are drawn at -0.3 (thin-dashed lines), $0.3 \mathrm{mJy} \mathrm{beam}^{-1}$, and the rest are spaced by a factor of 2 . The sensitivity $(1-\sigma)$ is $0.1 \mathrm{mJy}_{\text {beam }}^{-1}$. Crosses indicate the positions of the subtracted discrete sources. Arrows indicate hints of possible diffuse emission left after the subtraction process. Bottom (left): zoom of the "Main" cluster in which the total intensity radio contours are overlaid on the red plate of the Sloan Digital Sky Survey. Bottom (right): zoom of the "Main" cluster in which the total intensity radio contours are overlaid on the XMM X-ray image.

peripheral patch previously found by Venturi et al. (2008). In addition, the diffuse radio emission of the "Main" cluster is elongated toward the "Subcluster". As measured from the 3- $\sigma$ radio isophote, the overall diffuse emission has an angular extension of about $7^{\prime}(\simeq 1.8 \mathrm{Mpc}$ at the cluster distance).

To separate the diffuse radio emission from discrete sources we produced images at higher resolution. In the middle panel of Fig. 2 we present the radio iso-contours of A781, taken with the VLA in C configuration. This image has a $F W H M$ beam of $13.2^{\prime \prime} \times 16.5^{\prime \prime}$. Although the relatively high resolution of this image is not particularly suitable for detecting diffuse cluster emission, the peripheral patch of diffuse emission is clearly visible in the $\mathrm{C}$ array data-set too. The discrete sources are labeled in the figure and their position and flux densities are given in Table 2. All of them show an optical counterpart in the Sloan Digital Sky Survey (see bottom left panel of Fig. 3). The only exception is source B, which may be a background radio galaxy. Source D, which is located about 50" west of the X-ray peak of the "Main" cluster, is the brightest radio source in the field. Its fractional polarization is $\simeq 3 \%$. The other discrete sources embedded in the halo have a fractional polarization below the 3- $\sigma$ noise level. The
Table 2. Information on discrete radio sources.

\begin{tabular}{lccl}
\hline \hline Label & $\begin{array}{c}\text { RA } \\
(J 2000)\end{array}$ & $\begin{array}{c}\text { Dec } \\
(\text { J2000) }\end{array}$ & $\begin{array}{l}S_{1400 \mathrm{MHz}} \\
(\mathrm{mJy})\end{array}$ \\
\hline $\mathrm{A}$ & 092001.3 & 303405 & $21.1 \pm 0.6$ \\
$\mathrm{~B}$ & 092008.3 & 303215 & $14.9 \pm 0.5$ \\
$\mathrm{C}$ & 092014.0 & 302900 & $14.7 \pm 0.5$ \\
$\mathrm{D}$ & 092022.7 & 302945 & $67.0 \pm 2.0$ \\
$\mathrm{E}$ & 092021.9 & 303225 & $1.5 \pm 0.05$ \\
$\mathrm{~F}$ & 092048.6 & 302836 & $1.0 \pm 0.03$ \\
$\mathrm{G}$ & 092108.3 & 302926 & $3.9 \pm 0.1$ \\
$\mathrm{H}$ & 092112.7 & 302811 & $1.5 \pm 0.05$ \\
$\mathrm{I}$ & 092116.2 & 303031 & $20.7 \pm 0.6$ \\
$\mathrm{~L}$ & 092122.2 & 302911 & $2.5 \pm 0.08$ \\
\hline
\end{tabular}

Notes. Column 1: source label; Cols. 2, 3: source position (RA, Dec); Col. 4: source flux density at $1400 \mathrm{MHz}$.

peripheral diffuse emission appears unpolarized too, and given that its surface brightness is $\simeq 0.5 \mathrm{mJy}_{\text {beam }}^{-1}$, and the sensitivity (1- $\sigma$ ) of the linear polarization image is $\simeq 0.08 \mathrm{mJy} \mathrm{beam}^{-1}$, we 


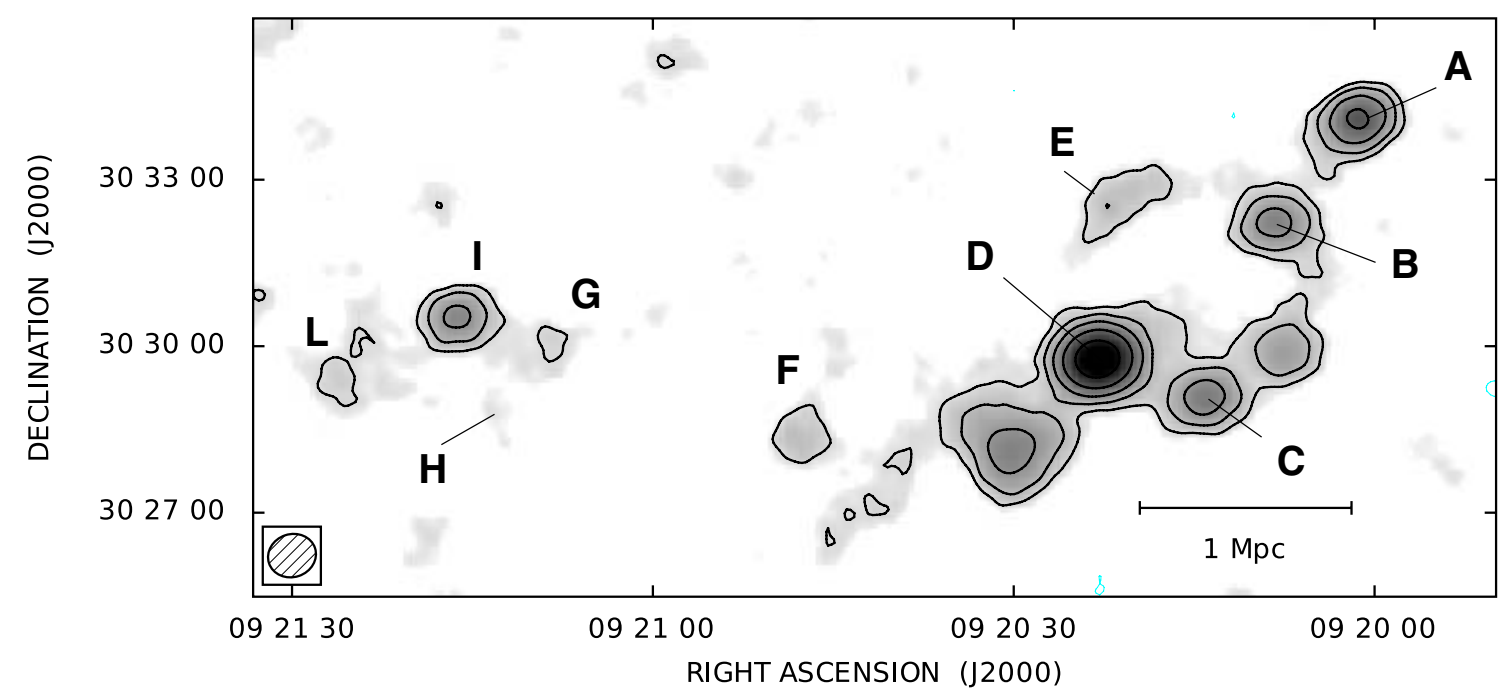

Fig. 4. Total intensity radio contours of the $\mathrm{A} 781$ system at $325 \mathrm{MHz}$ with the VLA in $\mathrm{B}+\mathrm{C}+\mathrm{D}$ configuration. The image has a $F W H M$ of $46.1^{\prime \prime} \times 52.0^{\prime \prime}\left(\mathrm{PA}=-71.8^{\circ}\right)$. The contour levels are drawn at -2.9 (thin-dashed lines), $2.9 \mathrm{mJy} \mathrm{beam}^{-1}$, and the rest are spaced by a factor of 2 . The sensitivity $(1-\sigma)$ of this image (not corrected for the primary beam) is 0.95 mJy beam ${ }^{-1}$. The image primary beam corrected has a sensitivity $(1-\sigma)$ of $2.2 \mathrm{mJy}$ beam $^{-1}$.

can just set a loose 3- $\sigma$ upper limit to the fractional polarization of $<48 \%$, consistent with the values found for other relic sources.

The image obtained with the VLA in A configuration has a $F W H M$ beam of $1.2^{\prime \prime} \times 1.4^{\prime \prime}$ and, in agreement with the $\mathrm{C}$ array configuration, confirms that just a few discrete sources are present in the field. Among the discrete sources, those labeled with A, B, C and D (shown in the bottom panel of Fig. 2) are extended. While A, C, and D show the typical morphology of "head-tail" radio sources in cluster, source B shows a straight "naked-jet" morphology.

The total flux density in the region of the "Main" and "Subcluster" is $\simeq 119 \pm 4 \mathrm{mJy}$. By subtracting the flux density of the embedded discrete sources C, D, and E as derived in the C configuration data-set (see Table 2), a flux density of $\simeq 36 \pm 5 \mathrm{mJy}$ appears to be associated with the low brightness diffuse emission. This flux density value corresponds to a radio power $P_{1400 \mathrm{MHz}}=1.0 \times 10^{25} \mathrm{~W} \mathrm{~Hz}^{-1}$. A781 is part of the ROSAT brightest cluster sample by Ebeling et al. (1998). Its $\mathrm{X}$-ray luminosity in the $0.1-2.4 \mathrm{keV}$ band is $1.1 \times 10^{45} \mathrm{erg} / \mathrm{s}$. The radio power $P_{1400 \mathrm{MHz}}$, the radio largest linear size $(L L S)$, and the X-ray luminosity $\left(L_{\mathrm{X}}\right)$ agree with the $P_{1400 \mathrm{MHz}}-L L S$ and $P_{1400 \mathrm{MHz}}-L_{\mathrm{X}}$ relations known for the other halos in clusters. The peripheral patch alone has a flux density $\simeq 15.5 \pm 0.5 \mathrm{mJy}$. Thus, if we consider the radio halo separated by the peripheral patch, it results in a flux density of $\simeq 20.5 \pm 5 \mathrm{mJy}$. This value corresponds to a radio power $P_{1400 \mathrm{MHz}}=5.9 \times 10^{24} \mathrm{~W} \mathrm{~Hz}^{-1}$, which still agrees with the $P_{1400 \mathrm{MHz}}-L L S$ and $P_{1400 \mathrm{MHz}}-L_{\mathrm{X}}$ relations known in the literature (see e.g. Giovannini et al. 2009).

The flux densities were calculated after the primary beam correction by integrating the total intensity surface brightness down to the $3 \sigma$ level. However, the residual flux density associated with the diffuse cluster emission must be interpreted with caution because of a possible variation in the discrete sources flux density, the slightly different frequency of the two data-sets, and any absolute calibration error between the two data-sets could cause in an under or over subtraction of flux. In addition, owing to the short exposure time of the archival observations, some diffuse emission could be missed. Therefore, a deep follow-up investigation would be necessary to ensure the recovery of the entire radio flux and to unambiguously separate the emission of the diffuse emission from that of the unrelated discrete sources.

To ensure that the large-scale diffuse emission is not caused by the blending of discrete sources, we present the total intensity radio contours at $1400 \mathrm{MHz}$ with the VLA in D configuration in the top panel of Fig. 3, after subtraction of discrete sources. To obtain this, we produced an image of the discrete sources by using only the longest baselines of the $\mathrm{D}$ configuration dataset, and uniform weighting. The clean components of this image were then subtracted in the uv-plane by using the AIPS task UVSUB. The image with the discrete sources subtracted confirms the presence of a low-surface brightness radio halo at the cluster center connected to a brighter patch of peripheral emission to the southeast.

In the bottom panel of Fig. 3 we show a zoom of the "Main" cluster in which the total intensity radio contours, after subtraction of discrete sources, are overlaid on the red image of the Sloan Digital Sky Survey (left) and on the XMM X-ray image (right). This figure also clearly shows that the elongation of the radio emission versus west coincides with the "Subcluster". This feature is visible at $1400 \mathrm{MHz}$ in the D configuration data-set only, while in the higher resolution images presented here and in the FIRST image (Becker et al. 1995), any discrete source seems to be present. But in this location a source that is classified as a discrete source is detected in GMRT images at $610 \mathrm{MHz}$ (Venturi et al. 2008) and at $327 \mathrm{MHz}$ (Giacintucci et al. 2010).

Hints of possible diffuse emission (indicated by arrows in the top panel of Fig. 3) that was left after the subtraction process are present to the east of the "Main" cluster. These diffuse radio emissions might trace the process of a large-scale structure formation, where cosmic shocks that originated in complex merger events are able to amplify magnetic fields and accelerate synchrotron electrons along a cluster filament, although a deeper observation is required to confirm the presence of these faint emissions.

\subsection{Cluster radio emission at $325 \mathrm{MHz}$}

In Fig. 4 we show the radio iso-contours of A781 at $325 \mathrm{MHz}$. This image was obtained by combining the VLA data in B, C, 

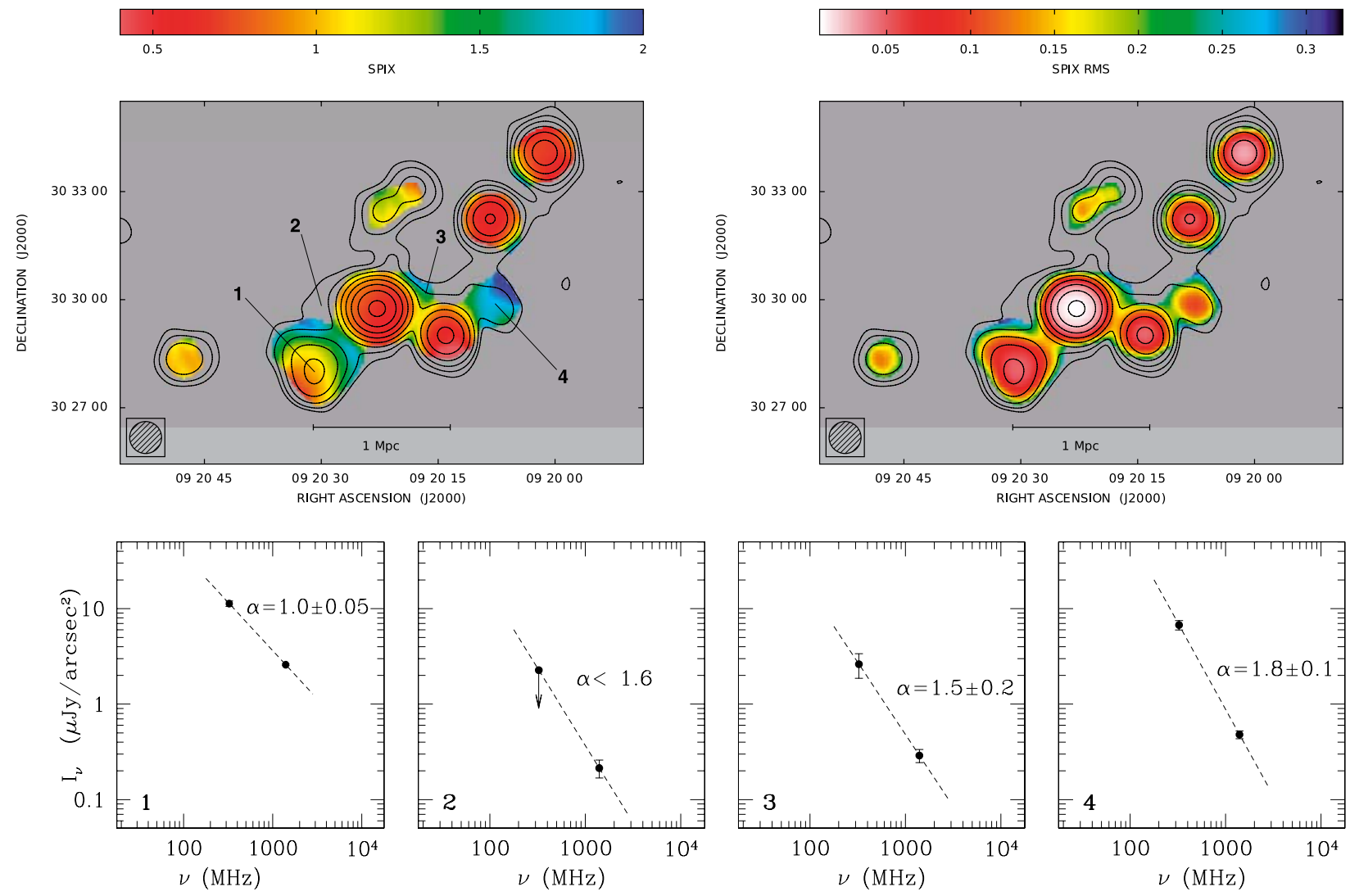

Fig. 5. Top left: spectral index image between 325 and $1400 \mathrm{MHz}$. The image has a $F W H M$ of $53^{\prime \prime} \times 53^{\prime \prime}$. The iso-contours represent the $1400 \mathrm{MHz}$ image convolved to a resolution of $53^{\prime \prime} \times 53^{\prime \prime}$ and corrected for the primary beam attenuation. The first contour level is drawn at $0.45 \mathrm{mJy}^{-1}$ beam ${ }^{-1}$ and the rest are spaced by a factor of 2 . The sensitivity $(1-\sigma)$ is $0.15 \mathrm{mJy}_{\text {beam }}{ }^{-1}$. The image at $325 \mathrm{MHz}$, convolved and primary beam corrected, has a sensitivity $(1-\sigma)$ of $2.5 \mathrm{mJy}_{\text {beam }}{ }^{-1}$. Top right: image of the spectral index uncertainty. Bottom: spectrum between $325 \mathrm{MHz}$ and $1400 \mathrm{MHz}$ in the four sample positions indicated in the top right panel.

and $\mathrm{D}$ configuration. The resulting image has a $F W H M$ beam of $46.1^{\prime \prime} \times 52.0^{\prime \prime}$. The VLA image has an angular resolution similar to the tapered GMRT image at $327 \mathrm{MHz}$ presented by Giacintucci et al. (2010), and displays the same structures.

Most of the features visible at $1400 \mathrm{MHz}$ are also present at $325 \mathrm{MHz}$. In particular, the southeast peripheral patch and all discrete sources are clearly detected with the only exception of the source $\mathrm{H}$. There is a hint of diffuse emission to the right of source $\mathrm{D}$, although most of the radio halo emission visible at $1400 \mathrm{MHz}$ is missing at $325 \mathrm{MHz}$, likely because of the lower sensitivity of the low-frequency image. The only feature that appears to be comparatively brighter at $325 \mathrm{MHz}$ is the emission that coincides with the "Subcluster". As we will see in the next section, this feature is characterized by a very steep radio spectrum.

If the peripheral patch is excluded, the radio halo has a flux density at $1400 \mathrm{MHz}$ of $S_{1400 \mathrm{MHz}} \simeq 20.5 \mathrm{mJy}$. In the same area of about $1 \mathrm{Mpc}^{2}$, the upper limit to the flux density at $325 \mathrm{MHz}$ is $S_{325 \mathrm{MHz}}<137 \mathrm{mJy}$. This limit was calculated by considering that the surface brightness of the halo in the primary beam corrected image is everywhere lower than the $3 \sigma$ noise level (i.e. $6.6 \mathrm{mJy} \mathrm{beam}^{-1}$ ). Thus, we derive an upper limit to the total halo spectral index of $\alpha_{\text {tot }}<1.3$.

\subsection{Spectral index analysis}

In this section we present the spectral index image of A781 between $325 \mathrm{MHz}$ and $1400 \mathrm{MHz}$. To facilitate a comparison the two images were corrected for the primary beam attenuation of the VLA, regridded to the same geometry, and convolved to a common resolution of $53^{\prime \prime} \times 53^{\prime \prime}$. We stress, however, that the original resolutions of the two images were already very close not just because of the tapering of the uv-data, but because of the very similar intrinsic coverage of the relevant spatial frequencies. We do not present the spectral index image after subtraction of discrete sources because of the low sensitivity of the $325 \mathrm{MHz}$ image.

In the top panel of Fig. 5 we present the spectral index (left) and the spectral index uncertainty (right) images between 325 and $1400 \mathrm{MHz}$ with the $1400 \mathrm{MHz}$ radio iso-contours (primary beam corrected) overlaid. They are calculated only from those pixels whose brightness is above the $3 \sigma$ level at both frequencies. The spectral index values range between $\alpha \simeq 0.5$ and $\alpha \simeq 2$, while the corresponding errors are in the range $\simeq 0.02-0.25$. The discrete sources have a typical spectral index value of $\alpha \simeq 0.6-0.7$, only the source $\mathrm{F}$ has a steep spectrum with $\alpha=1.0 \pm 0.2$. In the bottom panel of Fig. 5 we show the spectrum between $325 \mathrm{MHz}$ and $1400 \mathrm{MHz}$ in four sample positions.

The spectral index of the diffuse emission is clearly determined mainly in coincidence with the peripheral patch and the "Subcluster" (see positions 1 and 4 in Fig. 5). The peripheral patch shows a clear spectral index trend from $\alpha \simeq 0.9 \pm 0.1$ in the southeast tip to $\alpha \simeq 1.6 \pm 0.2$ close to the source $\mathrm{D}$. The radio elongation that coincides with the "Subcluster" has an extremely steep spectrum with $\alpha \simeq 1.8 \pm 0.1$, which gives an indication about the nature of this emission (see Sect. 5.2). 


\section{Discussion and conclusion}

Confirming the suggestion by Rudnick \& Lemmerman (2009), we find that the central region of the "Main" cluster of A781 is permeated by diffuse low-surface brightness radio emission, which is classified as a radio halo. The radio halo is clearly detected at $1400 \mathrm{MHz}$, while most of its emission is missing at $325 \mathrm{MHz}$, likely because of the lower sensitivity of the lowfrequency image. At $1400 \mathrm{MHz}$, the diffuse emission presents the typical extension and radio power of the other halos known in literature. In addition, two interesting features are found:

1) the radio halo is spatially connected to a peripheral diffuse patch, which was previously discovered by Venturi et al. (2008);

2) the cluster radio emission shows an elongation toward the "Subcluster".

\subsection{The peripheral diffuse patch}

The peripheral patch may be interpreted as part of the halo or as a radio relic (as suggested by Venturi et al. 2008; and Giacintucci et al. 2010). Radio relics are commonly defined as elongated diffuse cluster radio emission not associated with the cluster center (e.g. Giovannini \& Feretti 2004). They are usually interpreted as linked to the presence of shock waves that propagate in the intra-cluster medium during the process of cluster formation (e.g. Enßlin et al. 1998; Enßlin \& Gopal-Krishna 2001; Hoeft \& Brüggen 2007). Relic formation models predict a steepening of the radio spectral index toward the cluster center and a high degree of polarization (e.g. Clarke \& Enßlin 2006; Bonafede et al. 2009; van Weeren et al. 2010). For the peripheral patch emission of A781, the poor upper limit in the fractional polarization does not permit us to derive useful information. However, its location and its radio spectrum, which steepens toward the cluster center, may favor a relic origin for this emission.

\subsection{The radio emission of the "Subcluster"}

The diffuse radio emission of the "Main" cluster is elongated toward the "Subcluster" in a very similar way as the X-ray emission. The spectral index image reveals that the right end of this elongation has a very steep spectral index of $\alpha \simeq 1.8 \pm 0.1$. This emission seems to be really diffuse at $1400 \mathrm{MHz}$, because no point source is detected in this position either in the $\mathrm{C}$ array or in the A array image. Because this extreme steep spectrum is not compatible with an active galaxy, one possibility is that this may be a dying radio galaxy (e.g. Murgia et al. 2011). If this is the case, the optical progenitor could be the galaxy labeled $\mathrm{J}$ in the bottom left panel of Fig. 3. Venturi et al. (2008) found a faint radio counterpart associated to this galaxy with the GMRT at $610 \mathrm{MHz}$. Note, however, that the peak of the emission at $1400 \mathrm{MHz}$ is not precisely coincident with the galaxy J. Indeed, another possibility is that this emission is part of the radio halo or even a steep spectrum halo connected with the "Subcluster".

\subsection{Tracing the diffuse radio emission along a cluster filament}

In addition to the diffuse radio emission related to the "Main" and the "Subcluster", there are also some hints of diffuse emission elongated toward the east, on a larger scale. The nature of these faint patches, which are left after the subtraction of the discrete sources, is rather uncertain however in view of the current data. Nevertheless, if confirmed, the presence of these patches of diffuse emission may trace the process of a large-scale structure formation, where cosmic shocks originating in complex merger events are able to amplify magnetic fields and accelerate synchrotron electrons along a large-scale filament of the cosmic web.

Acknowledgements. We thank the anonymous referee for the suggestions that improved the presentation of the paper, and Luigina Feretti for the support and the helpful discussions. This research was partially supported by PRININAF 2009. The National Radio Astronomy Observatory (NRAO) is a facility of the National Science Foundation, operated under cooperative agreement by Associated Universities, Inc. Funding for the SDSS and SDSS-II has been provided by the Alfred P. Sloan Foundation, the Participating Institutions, the National Science Foundation, the US Department of Energy, the National Aeronautics and Space Administration, the Japanese Monbukagakusho, the Max Planck Society, and the Higher Education Funding Council for England. The SDSS Web Site is http://www.sdss.org/. This research made use of Montage, funded by the National Aeronautics and Space Administration's Earth Science Technology Office, Computational Technnologies Project, under Cooperative Agreement Number NCC5-626 between NASA and the California Institute of Technology. The code is maintained by the NASA/IPAC Infrared Science Archive.

\section{References}

Bacchi, M., Feretti, L., Giovannini, G., \& Govoni, F. 2003, A\&A, 400, 465 Becker, R. H., White, R. L., \& Helfand, D. J. 1995, ApJ, 450, 559

Bonafede, A., Giovannini, G., Feretti, et al. 2009, A\&A, 494, 429 Brunetti, G., Cassano, R., Dolag, K., \& Setti, G. 2009, A\&A, 507, 661 Buote, D. A. 2001, ApJ, 553, L15

Cassano, R., Ettori, S., Giacintucci, S., et al. 2010 ApJ, 721, L82

Clarke, T. E., \& Ensslin, T. A. 2006, AJ, 131, 2900

Cornwell, T. J., \& Perley, R. A. 1992, A\&A, 261, 353

Ebeling, H., Edge, A. C., Böhringer, H., et al. 1998, MNRAS, 301, 881

Enßlin, T. A., \& Gopal-Krishna 2001, A\&A, 366, 26

Enßlin, T. A., Biermann, P. L., Klein, U., \& Kohle, S. 1998, A\&A, 332, 395

Enßlin, T. A., Pfrommer, C., Miniati, F., \& Subramanian, K. 2011, A\&A, 527, A99

Feretti, L., Fusco-Femiano, R., Giovannini, G., \& Govoni, F. 2001, A\&A, 373, 106

Geller, M. J., Kurtz, M. J., Dell'Antonio, I. P., et al. 2010, ApJ, 709, 832

Giacintucci, S., Venturi, T., Brunetti, G., et al. 2005, A\&A, 440, 867 Giacintucci, S., et al. 2010, Presented at the NtGC2010 Conference, http://dl.dropbox.com/u/15734330/talks/giacintucci.pdf Giovannini, G., \& Feretti, L. 2000, New Astron., 5, 335

Giovannini, G., \& Feretti, L. 2004, J. Korean Astron. Soc., 37, 323

Giovannini, G., Tordi, M., \& Feretti, L. 1999, New Astron., 4, 141

Giovannini, G., Bonafede, A., Feretti, L., et al. 2009, A\&A, 507, 1257

Govoni, F., Feretti, L., Giovannini, G., et al. 2001a, A\&A, 376, 803

Govoni, F., Enßlin, T. A., Feretti, L., \& Giovannini, G. 2001b, A\&A, 369, 441

Govoni, F., Markevitch, M., Vikhlinin, A., et al. 2004, ApJ, 605, 695

Greisen, E. W., Spekkens, K., \& van Moorsel, G. A. 2009, AJ, 137, 4718

Hoeft, M., \& Brüggen, M. 2007, MNRAS, 375, 77

Jeltema, T. E., Canizares, C. R., Bautz, M. W., \& Buote, D. A. 2005, ApJ, 624, 606

Kempner, J. C., \& Sarazin, C. L. 2001, ApJ, 548, 639

Murgia, M., Govoni, F., Feretti, L., \& Giovannini, G. 2010, A\&A, 509, A86

Murgia, M., Parma, P., Mack, K.-H., et al. 2011, A\&A, 526, A148

Rengelink, R. B., Tang, Y., de Bruyn, A. G., et al. 1997, A\&AS, 124, 259

Rudnick, L., \& Lemmerman, J. A. 2009, ApJ, 697, 1341

Schuecker, P., Böhringer, H., Reiprich, T. H., \& Feretti, L. 2001, A\&A, 378, 408

Sehgal, N., Hughes, J. P., Wittman, D., et al. 2008, ApJ, 673, 163

van Weeren, R. J., Röttgering, H. J. A., Brüggen, M., \& Cohen, A. 2009, A\&A, 508,75

van Weeren, R. J., Röttgering, H. J. A., Brüggen, M., \& Hoeft, M. 2010, Science, 330,347

Venturi, T., Giacintucci, S., Brunetti, G., et al. 2007, A\&A, 463, 937

Venturi, T., Giacintucci, S., Dallacasa, D., et al. 2008, A\&A, 484, 327

Wittman, D., Dell'Antonio, I. P., Hughes, J. P., et al. 2006, ApJ, 643, 128 